Francesco S. Mennini

Laura Fioravanti

Laura Piasini

Fabio Palazzo

Gabriella Coloprisco

Paolo Martelletti

\section{A one-year retrospective economic evaluation of botulinum toxin type A treatment of chronic tension headache}

Received: 13 May 2004

Accepted in revised form: 15 September 2004

F.S. Mennini • L. Fioravanti • L. Piasini CEIS Centre for Health Economics and Health Care Management,

School of Economics,

University of Rome Tor Vergata,

Rome, Italy

\section{F. Palazzo}

National Research Council,

Institute for Population Research and Social Policies,

Rome, Italy

G. Coloprisco • P. Martelletti ( $($ 凶)

Department of Internal Medicine,

Regional Referral Headache Center,

2nd School of Medicine,

University of Rome La Sapienza,

Sant'Andrea Hospital,

Via di Grottarossa 1035,

I-00189 Rome, Italy

e-mail: paolo.martelletti@uniroma1.it

Tel.: +39-06-8034-5250/5684

Fax: +39-06-8034-5323

\begin{abstract}
The objective was to measure the impact of botulinum toxin type A (BTX-A) treatment on symptoms and medication utilisation patterns in patients with chronic tension-type headache. A retrospective chart analysis was completed in the Day Hospital of the Regional Referral Headache Centre at Sant'Andrea Hospital in Rome. Clinical charts were randomly selected for 100 patients treated from February 2002 to January 2003. Patients were treated with 100 U of BTX-A every three months for one year by using the Fixed Doses Fixed Sites procedure. Treatment outcome ranged from complete resolution of headache symptoms to a worsening of symptoms resulting in discontinuation. Headache medication use before and after treatment was analysed. After BTX-A treatment, $85 \%$ of patients experienced at least some degree of pain relief
\end{abstract}

and reduced their use of analgesics. The reduced percentages of patients using a variety of headache medications after BTX-A treatment results from a reduction in their headache symptoms.

Key words Chronic tension-type headache $\cdot$ Botulinum toxin type A

\section{Introduction}

Chronic tension-type headache usually evolves from the episodic form [1,2] and causes significant impairments in functioning. In one study, one-third of sufferers of chronic tension-type headache recorded impaired sleep, energy levels or emotional well-being on 10 days or more per month, and half of the patients exhibited clinically significant levels of anxiety or depression [3].

Botulinum toxin type A (BTX-A), a focally acting neurotoxin that inhibits the release of acetylcholine and other neurotransmitters from presynaptic nerve endings [4], represents a new therapeutic option for headache treatment. 
The results of several clinical trials suggest that BTX-A treatment significantly reduces the frequency, severity and disability associated with migraine and other types of headaches, with mixed results in the treatment of chronic tension-type headache [5-10]. Several clinical trials also report reduced headache analgesic use and expenditures following BTX-A treatment of migraine $[11,12]$. A recent open-label study showed that one year of BTX-A treatment for chronic tension-type headache reduced headache symptoms and decreased analgesic use and overall costs related to headache treatment [13].

The present evaluation was undertaken to increase our understanding of the pharmacoeconomic impact of BTX-A treatment of chronic tension-type headache.

\section{Materials and methods}

\section{Objective}

The purpose of this analysis was to measure the impact of BTX-A treatment of chronic tension-type headache on headache symptoms and patterns of medication utilisation.

\section{Design}

The study was divided into two parts: part one was a one-year retrospective analysis of headache medication utilisation patterns after BTX-A treatment of chronic tension-type headache based on data obtained from patients' clinical charts, and part two was a prospective analysis of real treatment costs before and after BTX-A treatment of the same patients otherwise not detectable from conventional clinical charts. BTX-A (100 units [U]) was administered every three months, at which time the treatment outcome was assessed and recorded. The Fixed Sites Fixed Doses (FSFD) procedure was closely followed in this study [13].

Participants

Clinical charts of chronic tension-type headache patients referred to the Day Hospital of the Regional Referral Headache Centre at the Sant'Andrea Hospital in Rome and treated from February 2002 to January 2003 were randomly selected. The study design has been approved by the Local Ethics Committee.

Diagnosis of chronic tension-type headache was made using the International Headache Society criteria [14]. Any patient whose charts indicated one of four headache treatment history profiles could participate. The profiles can be summarised as follows:

I. Patient used only one pharmaceutical for many years.

II. Patient consulted with only one specialist, had many modifications to therapy, and used only one pharmaceutical at a time.
III. Patient consulted with only one specialist, had many modifications to therapy, and simultaneously used a number of pharmaceuticals.

IV. The patient experienced many modifications to therapy, including the type of specialist, and simultaneously used more than one pharmaceutical.

All patients gave their written consent to participation in the study.

\section{Outcome measure}

The effects of BTX-A treatment were summarised by four general outcomes, ranging from complete resolution of headache symptoms to a worsening of symptoms that resulted in discontinuation of therapy, as summarised in Table 1 . These outcome profiles were arbitrarily designed for the purposes of this analysis to describe the varying degrees of efficacy as a result of BTX-A treatment. Visual analogue scale (VAS) and a daily headache diary have been additionally used to evaluate the effect of BTX-A treatment.

An analysis of headache medication utilisation described the percentages of patients using a particular class of medication before and after BTX-A treatment.

\section{Results}

Data were analysed on 100 chronic tension-type headache patients ( $81 \%$ female, mean age $40.7 \pm 11.6$ years). The patients' occupational status is shown in

Table 1 Treatment outcomes

\section{Treatment outcome type Description}

A

Total absence of pain, substantial

B reduction in the use of pharmaceuticals

C Significant reduction of pain, general reduction in the use of pharmaceuticals C Reduction of pain, slight reduction in D the use of pharmaceuticals No effect or "possible" worsening, no reduction in the use of pharmaceuticals

Table 2 Patient occupational profile

\begin{tabular}{lcc}
\hline Working activities & Male & Female \\
\hline Workers & 1 & 5 \\
Public salaried employees & 8 & 22 \\
Private salaried employees & 6 & 26 \\
Self-employed & 3 & 7 \\
Students & 1 & 3 \\
Housewife & 0 & 18 \\
Total & 19 & 81 \\
\hline
\end{tabular}


Table 2. Patients were assigned to previously established headache treatment history profiles as described in the Methods. Of 100 patients randomly selected, 16 belonged to Profile I, 27 to Profile II, 35 to Profile III and 22 to Profile IV (Fig. 1).

Apart from the 16 patients that followed only one type of therapy, the others (84) were divided as follows: 14

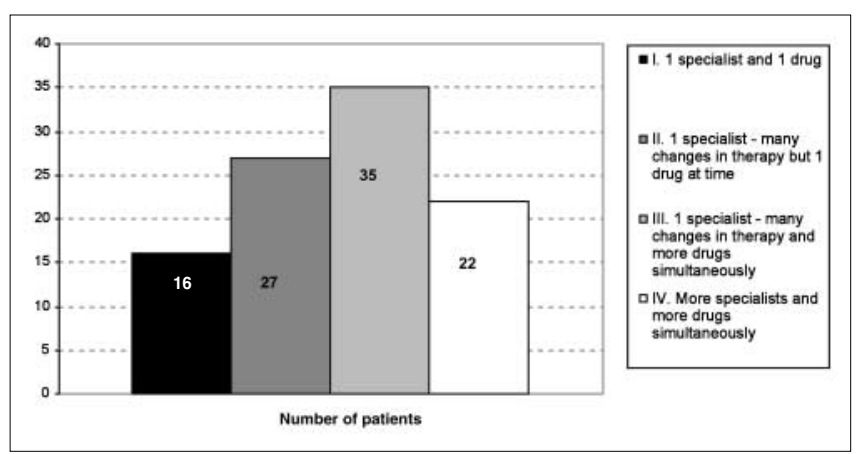

Fig. 1 Therapy profiles of chronic tension-type headache patients before using BTX-A

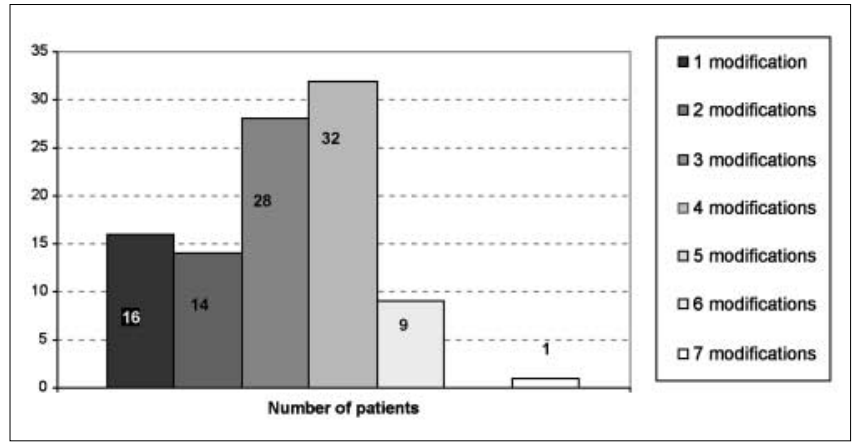

Fig. 2 Number of patients per treatment modification before using BTX-A patients had 2 headache treatment modifications (meaning they modified their initial therapy once); 28 patients had 3 modifications; 32 patients had 4 modifications; 9 patients had 5 modifications; no patients had 6 modifications; and, finally, 1 patient had 7 treatment modifications (Fig. 2).

The effect of BTX-A treatment identified 26 patients with total absence of pain and substantial reduction in the use of pharmaceuticals; 37 patients with significant reduction of pain and general reduction in the use of pharmaceuticals; 22 patients with reduction of pain and slight reduction in the use of pharmaceuticals; and 15 patients with no effect or "possible" worsening and no reduction in the use of pharmaceuticals (Fig. 3).

After one year of BTX-A treatment, the percentages of patients using calcium channel blockers fell from $76 \%$ to $0 \%$, barbiturate use fell from $49 \%$ of patients to $12 \%$, nonsteroidal anti-inflammatory drug (NSAID) use fell from $48 \%$ of patients to $32 \%$; antidepressants use fell from $24 \%$ of patients to $7 \%$; and antipyretics use fell from $7 \%$ of patients to $3 \%$ (Fig. 4).

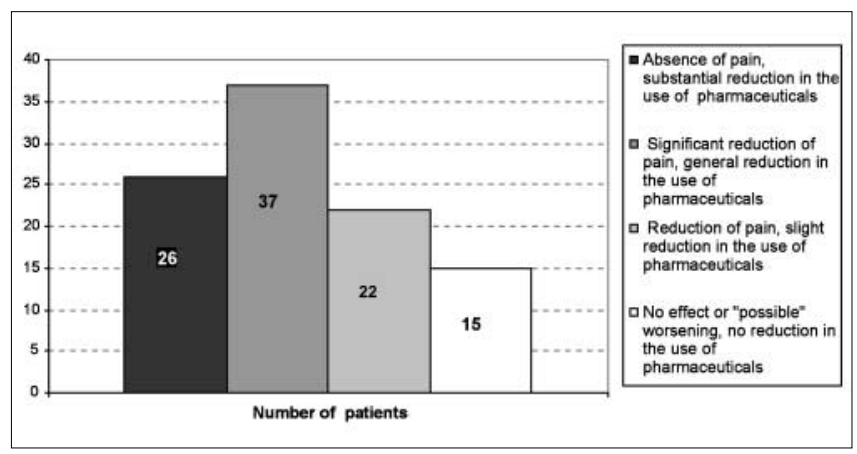

Fig. 3 The effects of BTX-A therapy
Fig. 4 Patients rate for single pharmaceutical category (before and after using BTX-A)

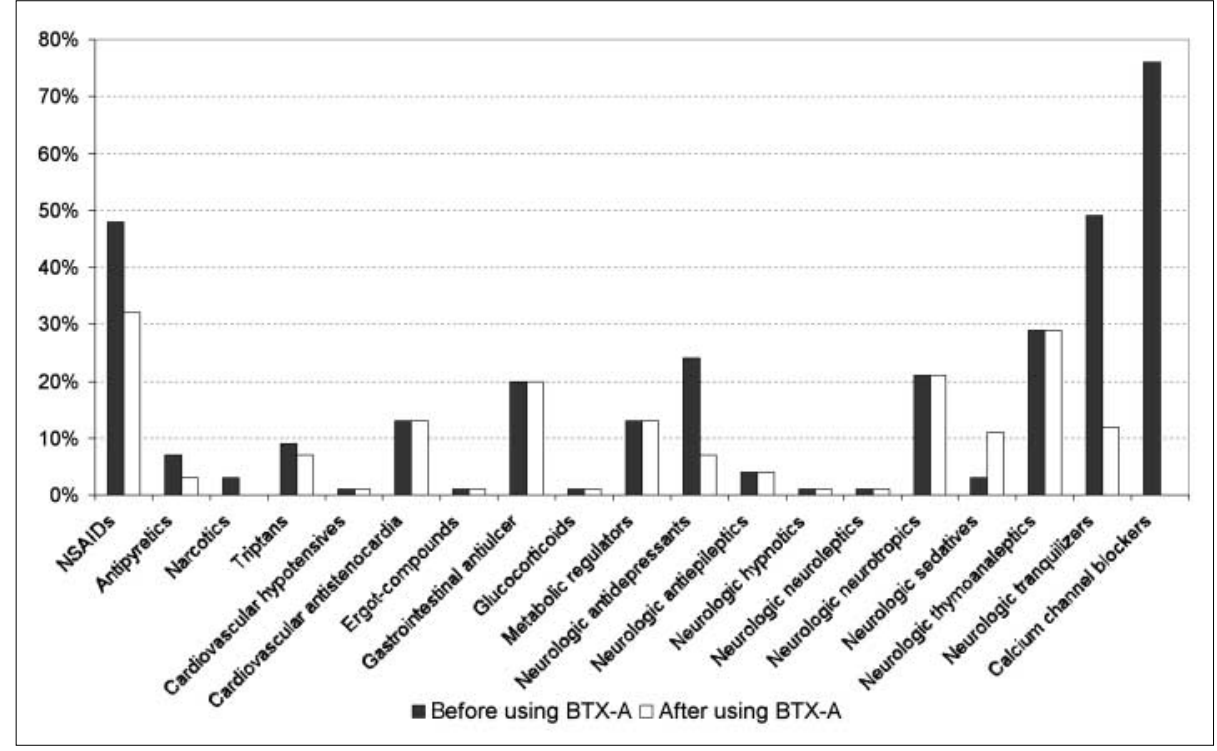




\section{Discussion}

The aim of this retrospective analysis was to find evidence of the efficacy of BTX-A in reducing the symptoms of chronic tension-type headache and, as a result, detect any changes in analgesic utilisation patterns after BTX-A treatment. We found that after BTX-A treatment, $85 \%$ of patients experienced at least some degree of pain relief and reduced their use of analgesics, especially calcium channel blockers, barbiturates, NSAIDs and antidepressants. A total of $63 \%$ of patients had either total or significant pain relief after BTX-A treatment and either a substantial or general reduction in analgesic use. A little more than a onequarter of all patients reported a total absence of pain after BTX-A treatment. Analgesic use reduced from a range of $7-76 \%$ of patients before BTX-A treatment to $0-32 \%$ of patients after BTX-A treatment.

The observed efficacy of BTX-A in reducing headache symptoms is consistent with the findings of previous clinical trials in a variety of headache types [5-9], and reduction of analgesic use after BTX-A treatment has also been previously observed $[8,11,12]$. Treatment with BTX-A appears to be a promising new therapeutic tool for chronic tension-type headache treatment.

However, interpretation of the results of this trial is limited by several factors, including the retrospective nature of the review. The observed changes in analgesic utilisation patterns were not quantified in terms of reductions in administered doses. In addition, the costs associated with analgesics or BTX-A injections were not considered. A prospective, pharmacoeconomic evaluation of BTX-A use in chronic tension-type patients conducted according to published guidelines for pharmacoeconomic evaluations in Italy $[15,16]$ was completed concurrently and is presented in part II of this analysis. The value of utilised resources (personnel, drugs, equipment and consumption of other resources for headache treatment), as well as the costs of administering BTX-A treatment, was incorporated.

Acknowledgements This research has been supported by an unrestricted educational grant from Allergan Inc, Irvine, USA.

\section{References}

1. Schwartz BJ, Stewart WF, Simon D, Lipton RB (1998) Epidemiology of tension-type headache. JAMA 279:381-383

2. Jensen K, Norup M (1992) Experimental pain in human muscle induced by hypertonic saline, potassium and acidity. Cephalalgia 12:101-106

3. Holroyd KA, Stensland M, Lipchik GL, Hill KR, O'Donnell FS, Cordingley G (2000) Psychosocial correlates and impact of chronic tensiontype headaches. Headache 40:3-16

4. Dolly O (2003) Synaptic transmission: inhibition of neurotransmitter release by botulinum toxins. Headache 43[Suppl 1]:16-24

5. Dodick DW (2003) Botulinum neurotoxin for the treatment of migraine and other primary headache disorders: from bench to bedside. Headache 43[Suppl 1]:25-33

6. Smuts J, Baker M, Smuts H (1999) Prophylactic treatment of chronic tension-type headache using botulinum toxin type A. Eur J Neurol 6[Suppl 4]:S99-S102
7. Wheeler AH (1998) Botulinum toxin A, adjunctive therapy for refractory headaches associated with pericranial muscle tension. Headache 38:468-471

8. Blumenfeld A (2003) Botulinum toxin type $A$ as an effective prophylactic treatment in primary headache disorders. Headache 43:853-860

9. Silberstein SD, Aoki KR (2003) Botulinum toxin type A: myths, facts and current research. Headache 43[Suppl 1]:1

10. Schmitt WJ, Slowey E, Fravi N, Weber S, Burgunder JM (2001) Effect of botulinum toxin A injections in the treatment of chronic tension-type headache: a double-blind, placebo-controlled trial. Headache 41:658-664

11. Schim J (2004) Effect of preventive treatment with botulinum toxin type A on acute headache medication usage in migraine patients. Curr Med Res Opin 20:49-53

12. Barrientos N, Chana P (2003) Botulinum toxin type $\mathrm{A}$ in prophylactic treatment of migraine headaches: a preliminary study. J Headache Pain 4:146-151
13. Coloprisco G, De Filippis S, Santi PG, Fiore G, Rodio A, Martelletti P (2003) Reduction in expenditure on analgesics during one year of treatment of chronic tension headache with BoNT-A. J Headache Pain 4:88-91

14. - (1998) Classification and diagnostic criteria for headache disorders, cranial neuralgias and facial pain. Headache Classification Committee of the International Headache Society. Cephalalgia 8[Suppl 7]:1-96

15. EURO-MED-STAT Group (2003) Monitoring expenditure and utilization of medicinal products in the European Union countries: a public health approach. Eur J Public Health 13[Suppl 3]:95-100

16. Capri S, Ceci A, Terranova L et al (2001) Guidelines for economic evaluations in Italy: recommendations from the Italian group of pharmacoeconomic studies. Drug Inf J 35:189-201 